\section{Herd protection or herding cats?}

\author{
Mark van der Linden, ${ }^{\oplus 1}$ Cynthia G Whitney ${ }^{2}$
}

In this issue of Thorax, Hanquet et $a l^{1}$ present results of surveillance for invasive pneumococcal disease (IPD) among adults aged 65 years and older in 10 European countries during the 5 years 2011-2015 following introduction of second-generation pneumococcal conjugate vaccines for infants that covered 10 (PCV10) or 13 (PCV13) of the more than 90 pneumococcal serotypes. They also compared the incidence rates to the pre-PCV10/13 year 2009. A total of 56194 cases of disease are reported. Since the authors are quantifying the effects of PCVs rolled out as part of infant immunisation programmes throughout Europe around 2010, the trends described are entirely indirect, or herd, effects. In other words, the authors describe how well pneumococcal conjugate vaccine programmes are interrupting pneumococcal transmission from children, and therefore, preventing disease in vulnerable older adults. The key questions the authors address are whether herd protection effects differ depending on the vaccine used in childhood immunisation programmes (PCV10 or PCV13) and whether vaccination of the elderly themselves with PCV13 has value, considering the reduction in circulating vaccine-serotype pneumococci and herd protection.

Given the high number of pneumococcal serotypes that have been described, trying to prevent overall disease using vaccines that target a relatively small number of serotypes could end up being like herding cats; disease caused by some serotypes will move in the right direction, and disease caused by others may not. Most countries in Europe started infant pneumococcal vaccination programmes between 2006 and 2009, using the first-generation vaccine that targeted the seven serotypes causing most serious disease (PCV7). These programmes were highly effective for preventing disease caused by the included serotypes, both with direct and indirect effects. ${ }^{2}$ However, in many countries disease caused by serotypes that PCV7

\footnotetext{
${ }^{1}$ National Reference Center for Streptococci, Department of Medical Microbiology, University Hospital RWTH Aachen, Aachen, Germany ${ }^{2}$ Centers for Diseases Control and Prevention, Atlanta, Georgia, USA
}

Correspondence to Dr Mark van der Linden, National Reference Center for Streptococci, Department of Medical Microbiology, University Hospital RWTH Aachen, Aachen 52074, Germany; mlinden@ukaachen.de did not cover became more common, with serotype $19 \mathrm{~A}$ in particular emerging as a key 'replacement' serotype. ${ }^{3}$

Both PCV10 and PCV13 have antigens targeting the same 10 serotypes, and PCV13 in addition has antigens that target serotypes 3, 6A and 19A. In the current analysis, the authors found that indirect effects in PCV10-using countries were of smaller magnitude than in PCV13 countries, and they suggest that this difference is driven by differences in indirect effects for serotype 19A specifically (174\% increase in PCV10-using countries vs $47 \%$ decrease in PCV13-using countries). Although PCV10 contains a $19 \mathrm{~F}$ antigen but no 19A antigen, other reports suggest that PCV10's 19F antigen produces cross-protective antibodies that may prevent 19A disease in vaccinated children. ${ }^{4}$ The data in the current article by Hanquet $e t a l^{2}$ provide evidence that any cross-protection from the $19 \mathrm{~F}$ antigen is not strong enough to reduce carriage and transmission of 19A strains. To judge whether PCV10 provides direct protection to $19 \mathrm{~A}$, more data might be needed. In this aspect, a recent correspondence from Belgium showing an increase in invasive disease caused by 19A among children after switching the national immunisation programme from PCV13 to PCV10 is of interest. ${ }^{5}$

The reduction in IPD in adults over 65 years of age in the 10 European countries is only $9 \%$ overall $(-14 \%$ in PCV13 countries, $-1 \%$ in PCV10 countries), but note that most countries contributing data to the analysis would have benefited earlier from indirect effects of their PCV7 programmes and any new indirect benefits from introduction of PCV10 or PCV13 would be limited to only the additional serotypes in the expanded-valent vaccines. The authors also noted very limited indirect effects on serotype 3 , even in those countries using the serotype 3 -containing PCV13. This is in accordance with data suggesting that the serotype 3 antigen may be less effective than the other vaccine-serotype antigens ${ }^{6}$ and may be insufficient to provide indirect protection. The small decrease in overall IPD shows that the reduction in vaccine type IPD in the 10 European countries through indirect effects is almost completely compensated by an increase in serotypes not included in the vaccines. In the USA, the situation is different, with a net reduction of adult IPD following introduction of PCV13 with no increase in non-vaccine type IPD to date. ${ }^{7}$ No one has explained what causes these differences in replacement disease observed between populations, but vaccine-induced shifts, natural secular trends and underlying population immunity may all play a role.

The current situation in Europe, with substantial pneumococcal disease burden among adults, and serotype 19A remaining prevalent especially in PCV10 using countries, suggests that direct vaccination of adults might be helpful. To be most effective, vaccines given to adults should not target disease caused by the same serotypes prevented most effectively by vaccinating children and stopping transmission. So far, the only conjugate vaccine licensed for adults has been PCV13, but new formulations (PCV15, PCV20) are in the pipeline. In addition to serotype 3 , serotypes such as 8 and $12 \mathrm{~F}$ have been found to be the most common in adults in recent publications $^{8-10}$ and currently in Germany the most prevalent adult serotypes are 3, 8, 9N, 22F and 12F (Mark van der Linden, personal communication). Therefore, it would seem important to include these serotypes in a new adult conjugate formulation. Concerning the choice of PCV for childhood programmes, the prevalence of $19 \mathrm{~A}$ will be the main consideration, especially if antibiotic-resistant strains are common.

The analysis has some limitations worth mentioning. Although 10 countries contributed to the surveillance programme, the majority of isolates (55\%) were from England and Wales and from France, countries that exclusively use PCV13. The data assessing impact of PCV10 are therefore much less robust than data from PCV13-using countries, and conclusions should be viewed in that context. Nonetheless, this paper makes an important contribution in that it attempts to directly compare PCV10 programme benefits to PCV13 programme benefits. To our knowledge, only one other publication has presented a side-by-side analysis of programme impacts linked to routine use of the two vaccines, a study from Sweden that compared disease trends in counties providing PCV10 compared with those that vaccinated children with PCV13. ${ }^{11}$ Similar to findings presented here, the investigators in Sweden noted larger benefits in counties using PCV13, in large part because of differences in effects on disease caused by serotype 19A.

The authors here found that overall herd effects among older adults-a 
combination of reductions in vaccine serotypes and increases in serotypes that the vaccines do not target-differed somewhat according to the PCV product used in the participating countries' childhood vaccination programmes. Importantly, they show that the overall IPD incidence among adults over 65 years of age did not decrease to a large degree after introduction of second-generation PCVs targeting a few more serotypes, after accounting for replacement disease. The findings show, once again, that some pneumococcal serotypes do not cooperate with attempts at disease prevention-more like herding cats than protecting the herd.

Funding The authors have not declared a specific grant for this research from any funding agency in the public, commercial or not-for-profit sectors.

Competing interests None declared.

Patient consent for publication Not required.

Provenance and peer review Commissioned; externally peer reviewed.

Data sharing statement Requested comment.

C Author(s) (or their employer(s)) 2019. No commercial re-use. See rights and permissions. Published by BMJ.

D Check for updates
To cite van der Linden M, Whitney CG. Thorax 2019;74:425-426.

Accepted 11 February 2019

Published Online First 6 March 2019

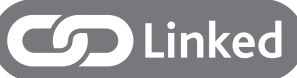

http://dx.doi.org/10.1136/thoraxjnl-2018-211767

Thorax 2019;74:425-426.

doi:10.1136/thoraxjnl-2018-212585

\section{REFERENCES}

1 Hanquet G, Krizova P, Valentiner-Branth P, et al. Effect of childhood pneumococcal conjugate vaccination on invasive disease in older adults of 10 European countries: implications for adult vaccination. Thorax 2019:74:473-82.

2. Feikin DR, Kagucia EW, Loo JD, et al. Serotype-specific changes in invasive pneumococcal disease after pneumococcal conjugate vaccine introduction: a pooled analysis of multiple surveillance sites. PLoS Med 2013;10:e1001517.

3. Miller E, Andrews NJ, Waight PA, et al. Herd immunity and serotype replacement 4 years after seven-valent pneumococcal conjugate vaccination in England and Wales: an observational cohort study. Lancet Infect Dis 2011:11:760-8.

4. Jokinen J, Rinta-Kokko H, Siira L, et al. Impact of ten-valent pneumococcal conjugate vaccination on invasive pneumococcal disease in Finnish children--a population-based study. PLoS One 2015;10:e0120290.
5. Desmet S, Verhaegen J, Van Ranst M, et al. Switch in a childhood pneumococcal vaccination programme from PCV13 to PCV10: a defendable approach? Lancet Infect Dis 2018;18:830-1.

6. Sings $\mathrm{HL}$, De Wals P, Gessner BD, et al. Effectiveness of 13 -Valent pneumococcal conjugate vaccine against invasive disease caused by serotype 3 in children: a systematic review and meta-analysis of observational studies. Clin Infect Dis 2018.

7. Moore MR, Link-Gelles R, Schaffner W, et al. Effect of use of 13-valent pneumococcal conjugate vaccine in children on invasive pneumococcal disease in children and adults in the USA: analysis of multisite, population-based surveillance. Lancet Infect Dis 2015:15:301-9.

8. Ladhani SN, Collins S, Djennad A, et al. Rapid increase in non-vaccine serotypes causing invasive pneumococcal disease in England and Wales, 2000-17: a prospective national observational cohort study. Lancet Infect Dis 2018;18:441-51.

9. van der Linden M, Falkenhorst G, Perniciaro S, et al. Effects of infant pneumococcal conjugate vaccination on serotype distribution in invasive pneumococcal disease among children and adults in Germany. PLOS One 2015;10:e0131494.

10. Hausdorff WP, Hanage WP. Interim results of an ecological experiment - Conjugate vaccination against the pneumococcus and serotype replacement. Hum Vaccin Immunother 2016;12:358-74.

11. Naucler P, Galanis I, Morfeldt E, et al. Comparison of the impact of pneumococcal conjugate vaccine 10 or pneumococcal conjugate vaccine 13 on invasive pneumococcal disease in equivalent populations. Clin Infect Dis 2017;65:1780-90. 